\title{
Pobreza y funcionamiento psicológico. Revisión sucinta de una relación en dos vías
}

\section{Poverty and psychological functioning. Brief review of a two-way relation}

DOI: http://dx.doi.org/10.17981/cultedusoc.8.1.2017.05

Suelen Emilia Castiblanco-Morenoํㅜ e Iván Felipe Medina-Arboleda ${ }^{2}$

\begin{abstract}
Resumen
Los estudios acerca de la pobreza y sus efectos sociales, políticos, culturales y económicos han ocupado un lugar central en las ciencias sociales. La combinación de los campos de conocimiento de la Psicología y la Economía han hecho grandes contribuciones a dilucidar sus relaciones. El propósito de esta revisión sucinta es sintetizar las tendencias conceptuales de la psicología cognitiva sobre la pobreza como fenómeno social en dos versiones: (a) la pobreza determinada por aspectos psicológicos del decisor como enfoque primigenio al problema y (b) la afectación de las condiciones de pobreza al funcionamiento psicológico como visión contemporánea que inspira la acción disciplinar y política multilateral. Se discuten los hallazgos sobre afectación psicológica que dieron lugar al cambio en la política económica mundial de lucha contra la pobreza y las implicaciones metodológicas y teóricas para la psicología de asumir la pobreza como condición de decisión y no como característica de la muestra.
\end{abstract}

Palabras clave: Psicología cognitiva; economía del comportamiento; pobreza; marcos de decisión; teoría del prospecto.

Recibido: Febrero 15 de 2017 / Aceptado: Abril 24 de 2017

\footnotetext{
${ }^{1}$ Docente de la Facultad de Ciencias Económicas y Sociales, Universidad de la Salle, Colombia. Correspondencia: secastiblanco@unisalle.edu.co

${ }^{2}$ Profesor del programa de Doctorado en Psicología, Universidad Católica de Colombia, Colombia. Correspondencia: ifmedina@ucatolica.edu.co
} 


\begin{abstract}
Studies of poverty and its social, political, cultural and economic effects have occupied a central place in the social sciences. The combination of the fields of knowledge of Psychology and Economics have made great contributions to elucidate their relationships. The purpose of this brief review is to summarize the conceptual trends in cognitive psychology on poverty as a social phenomenon in two trends: (a) poverty determined by psychological aspects of the consumer as first approach to the problem, and (b) the impact of the conditions from poverty to psychological functioning as a contemporary vision that inspires disciplinary action and multilateral politics. Findings on psychological effects that led to the change in global economic policy to fight against poverty and methodological and theoretical psychology to take poverty as a condition decision and not as characteristic of the sample implications are discussed.
\end{abstract}

Keywords: Cognitive psychology; behavioral economics; poverty; decision frames; prospect theory.

\title{
Introducción
}

Desde el inicio mismo de la ciencia económica, la búsqueda por solucionar el problema de la pobreza ha sido, en palabras de Banerjee, Benabou y Mookherjee (2006, pág. 20) “(...) uno de los más sagrados de los Santos Griales”, esto se traduce en un gran abanico de posturas sobre sus causas. En años recientes, la perspectiva económica más ortodoxa ha centrado la discusión sobre cómo combatir esta problemática, hacia la importancia de hacer reformas políticas e institucionales, mejorar las redes de seguridad social, construir escuelas para los más pobres, crear impuestos progresivos, promover la democracia, asegurar el cumplimiento de la ley, reforzar el rol de la población civil, entre otras.

Para Banerjee, et al (2006), esta postura no es más que la actualización de las visiones tradicionales en economía, en las que, por un lado, se cuentan quienes consideran que hacer algo para resolver la pobreza es "(...) infructuoso debido a que su base está en características inmutables de personalidad, cultura o geografía." (pág. 21), o lo que se conoce de forma común como "cultura de pobreza", en la que la cultura de pobreza es entendida como un conjunto de valores que es adaptativo en condiciones de pobreza, pero limitante al final (Dornbusch \& Edwards, 1991; Katz, 1989; Spears, 2011). Por otro lado, están los defensores del Consenso de Washington que piensan que el crecimiento económico es el principal determinante de la pobreza, y que la única solución son políticas orientadas hacia la liberalización de los mercados que aumenten el Producto Interno Bruto de los países dado que, -dar tajadas más grandes a cada persona, solo es posible si hay torta más grande - (Aghion \& Armendáriz, 2006; Graham, 1994)

Una tercera visión, con menor tradición y altas influencias de la teoría del desarrollo humano (Sen, 2015), defiende la idea de que las raíces de la pobreza tienen una estrecha relación con la desigualdad en la distribución de los recursos y con el diseño de las instituciones coloniales (África, América Latina y Sureste Asiático). Desde esta perspectiva, para mejorar las condiciones de las personas pobres se requiere de la ayuda internacional, de modo tal que estos flujos de recursos permitan hacer las inversiones necesarias y generen el impulso requerido para alcanzar los estándares de vida de los países más desarrollados (Acemoglu \& Johnson, 2006; Sachs, 2006). 


\section{Psicología y pobreza: Caracterización de la relación}

De acuerdo con Turner y Lehning (2007), desde la segunda mitad del siglo XX, los psicólogos empezaron a preocuparse por las personas en esta situación de pobreza, así, las teorías explicativas ubicaron el origen de la pobreza en los individuos o en la cultura, pero no en las fuerzas sociales y estructurales que pueden afectarla.

El primer conjunto de teorías se basó en las que en su momento eran las recién construidas pruebas para medir la inteligencia. Una de las teorías iniciales que intentó explicar la existencia y persistencia de la pobreza, se conoce como la perspectiva naturalizadora (naturalizing perspective) o nativista, que considera que los factores biológicos intrínsecos (de forma puntual bajos desempeños en las mediciones de inteligencia) son los que conducen a la pobreza (Rainwater, 1970 citado por Turner \& Lehning, 2007). Una teoría relacionada, sostiene que un desarrollo tardío del lenguaje sumado a un ambiente empobrecido, llevan a un pobre desempeño académico y a la perpetuación de la pobreza (Pearl, 1970 citado por Turner \& Lehning, 2007).

Una de las teorías psicológicas con mayor respaldo, sobre las causas de la pobreza es el Enfoque McClelland, que sugiere que las personas en situación de pobreza no desarrollaron un rasgo de personalidad conocido como Necesidad de logro, lo que les impide tomar acciones para mejorar su situación (McClelland, 1978). Ya en la década de 1980, desde la teoría de la atribución se planteó que los individuos pobres tendían a atribuir sus fracasos a factores internos, y sus éxitos a factores externos e incontrolables. Finalmente, es posible encontrar otras teorías psicológicas que apuntan a que la pobreza es una manifestación de: deficiencias morales, enfermedades mentales, conductas pecaminosas, entre otras (Turner \& Lehning, 2007).

A la par de estos trabajos, y con la consolidación de la labor social del psicólogo, surgieron teorías orientadas a explicar la alta dependencia de las personas en situación de pobreza de los servicios estatales, así se posicionaron un conjunto de explicaciones de corte psicoanalítico que identificaron el desarrollo psicosexual y el ego como causas de desórdenes de carácter que podrían perpetuar la pobreza (Curran, 2002).

Carr et al, (2014) señalan que las décadas de 1970 y 1980 se caracterizaron por una especie de crisis de confianza en la habilidad de la psicología para aportar a la solución de la pobreza. En ese sentido, no sorprende entonces que la verdadera revolución haya provenido del trabajo interdisciplinario entre la economía y la psicología, cuando, inicialmente por parte de un economista, Herbert Simon, y años después con el trabajo de dos psicólogos, Amos Tversky y Daniel Kahneman, surgiera un campo de estudio que transformó de forma definitiva la manera en que las ciencias sociales se aproximan a diferentes fenómenos, particularmente a aquellos relacionados con la elección de los individuos.

Herbert Simon, planteó en 1947 la teoría de la racionalidad limitada en la que incluyó tres elementos claves a saber: primero, el papel de la incertidumbre y el riesgo como determinantes de la toma de decisiones, segundo, la noción de información asimétrica en cuanto a que los individuos solo tienen acceso y pueden procesar una cantidad limitada de información, y tercero, la complejidad existente al evaluar los costos de las opciones u otras restricciones ambientales que pueden impedir al agente calcular el mejor curso de acción (Simon, 1972). 
Años después, Daniel Kahneman y Amos Tversky retomaron la teoría de la racionalidad limitada con el fin de construir un mapa que involucrara la influencia que tienen los sesgos sistemáticos de los individuos en sus decisiones. Para Kahneman (2003) esta aproximación a la toma de decisiones tenía un fin psicológico con posibles aplicaciones al campo económico, solo años después fue evidente que su trabajo había traspasado las barreras de lo disciplinario y había puesto la piedra angular para un nuevo campo de conocimiento: la economía del comportamiento (Behavioral Economics en idioma inglés).

La "Economía del Comportamiento (O conductual)" hace referencia a un campo de conocimiento interdisciplinario entre la psicología y la economía, que se caracteriza por cuatro líneas principales. "Primero, identificar hipótesis o modelos normativos que son usados por los economistas, tales como los modelos bayesianos, utilidad esperada y utilidad descontada. Segundo, identificar anomalías, por ejemplo, demostrar claras violaciones de las hipótesis o modelos y proponer cuidadosamente explicaciones alternativas (tales como la confusión de las personas sobre los costos de transacción). Y tercero, utilizar las anomalías como una inspiración para crear teorías alternativas que generalicen los modelos existentes. Un cuarto paso es la construcción de modelos de comportamiento usando las hipótesis conductuales del tercer paso, derivar nuevas implicaciones, y evaluarlas." (Camerer \& Loewenstein, 2004, pág. 6)

En el marco de la Economía del comportamiento, los aportes de Kahneman y Tversky constituyen un conjunto de tres programas de investigación diferentes pero interconectados conocidos como la Teoría de la prospectiva. El primero de estos, se orientó a explorar los heurísticos y acciones adicionales que toman los individuos cuando deben hacer una elección en un entorno de incertidumbre, el segundo, buscó desarrollar un modelo de elección en contextos con y sin riesgo, involucrando la aversión a la perdida en el modelo eleción, y el tercero se ocupó de los framing effects (Efectos marco) y el efecto de la presentación de las opciones en la elección de los individuos, y sus implicaciones para el modelo de agente racional egoísta, maximizador de ganancias y minimizador de costos (Kahneman, 2003).

La Teoría de la prospectiva, como forma simplificada de referirse a la arquitectura conceptual del modelo de Kahneman y Tversky, parte de un concepto presente en el modelo de atención limitada de Kahneman (1973). De acuerdo con este modelo, existe una estructura formada por dos sistemas, un Sistema 1 o intuitivo que ejecuta respuestas rápidas, en paralelo con otras respuestas, automáticas, emocionales, que no generan una gran demanda de recursos atencionales, y difícilmente controlables y modificables por estar asociadas con la costumbre. Por su lado, el Sistema 2 realiza operaciones calculadas, controladas, más lentas, con alta demanda de recursos y regidas por reglas lógicas. En síntesis, la interacción entre estos dos sistemas puede plantearse como que "el Sistema 2 controla las actividades del sistema 1" (Kahneman, 2003, pág. 185).

La teoría clásica de la decisión considera, desde esta perspectiva, que las respuestas del agente tomador de decisiones son atribuibles al Sistema 2, en la medida en que son racionales. Sin embargo, Kahneman y Tversky lograron demostrar que varias de las suposiciones de la teoría clásica eran erradas. En primer lugar, encontraron que las elecciones no dependen de forma exclusiva de la utilidad concreta asociada a cada elección, sino que, en contextos de incertidumbre, se relaciona más con las variaciones de la utilidad generadas por cambios en las dotaciones de bienes o servicios de los individuos (Kahneman \& Tversky, 1979). 
En segundo lugar, Kahneman y Tversky probaron que las preferencias (predictores de la elección en el modelo de la teoría clásica) se ven afectadas por las variaciones en la descripción de las alternativas a las que se enfrentan los individuos al modificar la notabilidad de unos aspectos por encima de otros, este concepto fue definido como el framing effect (Kahneman, 2003).

En tercer lugar, Kahneman y Tversky propusieron una teoría de la elección alternativa a la teoría clásica. Identifican un sistema cognitivo compuesto por una estructura racional, pero también una intuitiva que ofrece respuestas en apariencia lógicas ante situaciones que requieren de elecciones rápidas. Esto quiere decir que la intuición y el razonamiento son caminos alternativos para la solución de problemas, y que, con base en la minimización de costos en términos de actividades cognitivas, los agentes buscan simplificar el proceso de toma de decisiones, con lo que procesan de forma más superficial la información, son más susceptibles a construcciones que se basan en estereotipos y a la presentación de la información. En últimas, los agentes no toman decisiones mal razonadas sino decisiones intuitivas, que no se guían por los cálculos de los agentes sino por los elementos que perciben (Kahneman, 2003).

El llamado de atención de la psicología respecto al papel que juegan los elementos cognitivos y emocionales en la toma de decisiones produjo una transformación profunda en la ciencia económica. Thaler (2000) asegura que la revolución comportamental iniciada por Simon, está haciendo que la economía cambie su sujeto de estudio del Homo Economicus al Homo Sapiens, es decir, a un agente que aprende más despacio, con niveles de racionalidad diferentes, más emocional, entre otros. Esto implica que la ciencia económica debe empezar a estudiar la cognición humana y pasar de modelos normativos (lo que debe ser) a algunos más descriptivos (lo que es).

Este nuevo escenario permite comprender por qué son cada vez más frecuentes las aproximaciones cognitivas a temas económicos tan variados como las finanzas comportamentales, decisiones financieras de los individuos (Shefrin, 2002), hasta cómo estructurar reglas legales que tengan en cuenta la racionalidad limitada de los agentes (Sunstein, 2000). En la actualidad campos interdisciplinarios como las políticas públicas y la economía del desarrollo, también han empezado a utilizar este enfoque para explicar diversos fenómenos.

Así pues y como se mencionó previamente, el surgimiento de la economía comportamental está generando cambios en la forma en que las ciencias sociales estudian la pobreza, desde este campo de estudio se arguye que las personas en situación de pobreza están expuestas a los mismos sesgos en la toma de decisiones que quienes no están en esta situación, sin embargo, dado su estrecho margen de error, las equivocaciones pueden llevarlos a peores resultados. Por tanto, cualquier individuo, en una situación de carga cognitiva y marco de elección de riesgo tomará decisiones contraproducentes. De acuerdo con esto, se hace necesaria una aproximación más realista a la pobreza y un cambio en las políticas orientadas a su reducción (Bertrand, Mullainathan, \& Shafir, 2004).

En esta vía, en los últimos años han aumentado el número de investigaciones que abordan la pobreza desde un enfoque comportamental, (Banerjee \& Duflo, 2007; Banerjee, Duflo, Glennerster, \& Kinnan, 2013; Banerjee \& Mullainathan, 2010; Bertrand, Mullainathan, \& Shafir, 2006; Duflo, 2006; Shah, Mullainathan, \& Shafir, 2012). En un influyente artículo publicado en la revista Science, Mani, Mullainathan, Shafir y Zhao (2013) plantean la hipótesis de que las personas en situación de pobreza se comportan de 
maneras "menos capaces", lo que perpetúa su situación económica. En este trabajo, los investigadores, parten de la hipótesis de que tomar decisiones en un contexto de restricciones presupuestales deja menos recursos cognitivos disponibles para elegir y actuar.

Con el fin de evaluar esta hipótesis, realizaron dos estudios. El primero estuvo compuesto por cuatro experimentos con compradores en un centro comercial en Nueva York, cuyos ingresos variaban entre USD $\$ 20.000$ (pobreza) y USD $\$ 70.000$ (no pobreza) anuales. En uno de los experimentos, se les presentaron a los participantes, divididos de forma aleatoria en dos condiciones financieras, una "Dura" (costos altos) o "Fácil" (costos bajos), cuatro escenarios (con algunos minutos de diferencia) que describían un problema financiero. Después de ver cada escenario y pensar en cómo lo resolverían, los participantes debían realizar dos tareas para medir su función cognitiva (Test de Stroop y la Prueba de matrices de Raven). Como se esperaba, en la condición "Fácil" no hubo diferencias significativas en los desempeños de las tareas entre personas no pobres y pobres. Pero, en la condición "Dura”, las personas pobres tuvieron resultados mucho peores en las dos tareas, en comparación con las personas no pobres (Mani et al, 2013).

En el segundo estudio, participaron 464 cultivadores de caña de azúcar en India. Los individuos fueron entrevistados antes y después de la cosecha con lo que se identificó que enfrentan mayores presiones financieras antes que después de la cosecha. Al utilizar el Test de Stroop y la Prueba de Matrices de Raven para evaluar desempeño cognitivo, no solo encontraron los mismos resultados que en el estudio anterior - peores desempeños en las pruebas antes de la cosecha -, sino que hallaron correlaciones negativas entre la "intensidad de la restricción presupuestal percibida" por parte de los campesinos y sus resultados en la prueba de Raven. Además, en este segundo experimento, se registró el estrés (tasa cardiaca y presión sanguínea) antes y después de la cosecha, para examinar su relación con el desempeño en las pruebas cognitivas. En todos los análisis, corrigiendo los modelos por este factor, los resultados siguieron siendo significativos, lo que indica que el estrés no explica las diferencias obtenidas. (Mani et al, 2013; Mullainathan \& Shafir, 2016).

El trabajo de Mani, et al, (2013), dio el empuje que se requería a los estudios en pobreza desde la perspectiva comportamental. Hall, Zhao y Shafir (2013) identificaron que el estigma de pobreza puede llevar a una disminución del desempeño cognitivo y mayor distancia cognitiva, pero estos efectos pueden reducirse a través de procesos de autoafirmación que reduzcan el estigma. Haushofer y Fehr (2014), plantean que la situación de pobreza causa estados afectivos negativos que pueden llevar a un proceso de toma de decisiones corto de visión, adverso al riesgo, que limita la atención y favorece los comportamientos habituales en lugar de aquellos que se orientan al logro de objetivos.

Por su parte, Darminger, Hayes, Barrows y Wright (2015), señalan que las consecuencias cognitivas de vivir en situación de pobreza pueden ser benéficas o adaptativas en el corto plazo, pero muy perjudiciales si se convierten en regulares en el largo plazo. En el mismo sentido, Shah, Shafir y Mullainathan (2015) muestran que en condiciones de escasez las preferencias de las personas son moldeadas por los sacrificios a los que se enfrentan.

La explosión de investigaciones sobre la pobreza también es evidente en el campo de la psicología cognitiva y la neuropsicología. Por ejemplo, Lipina y Posner (2012) mencionan que las disparidades en el estatus socio económico (SES por sus siglas en inglés) afectan de forma negativa procesos cognitivos tales como: el lenguaje, las funciones ejecutivas, la 
atención y la memoria. Los autores presentan los hallazgos de cómo el sistema prefrontal/ejecutivo es uno de los que mayores afectaciones sufre por la disparidad en el ingreso en edades tempranas, lo que explicaría el bajo desempeño en pruebas cognitivas de los niños en situación de pobreza.

Asimismo, Calvo y Bialystok (2014), a partir de los hallazgos en una muestra de 175 niños de seis años divididos entre "clase media" y "clase trabajadora", encontraron que los niños de clase media tuvieron mejor desempeño que los de clase trabajadora en todas las otras pruebas (vocabulario - imagen, atención visual verbal y no verbal, control cognitivo y atención focalizada). Finalmente, Katsnelson (2015) con base en los trabajos de Noble, Norman y Farah (2005) y Raizada, Richards, Meltzoff, y Kuhl (2008), muestra que hay diferencias en el desarrollo cerebral del hipocampo y lóbulo frontal entre niños pobres y no pobres, así como déficits en los circuitos asociados a lenguaje, ciertas dimensiones de la memoria, y la habilidad para regular pensamientos y emociones.

En este contexto de hallazgos sobre las relaciones entre pobreza y funcionamiento psicológico, en un reconocimiento a los hallazgos interdisciplinares, en el 2015 el Banco Mundial publicó el "Reporte sobre el Desarrollo Mundial: Mente, Sociedad y Comportamiento", cuyo mensaje principal, en palabras de Jim Yong Kim, es que: "Al referirse a la comprensión y el cambio del comportamiento humano, podemos hacerlo mejor. Muchos economistas y profesionales del desarrollo creen que los elementos "irracionales" de la toma de decisiones humana son inescrutables o que se cancelan entre ellos cuando los grandes números de las personas interactúan, como en los mercados. Pero, nosotros sabemos ahora que ese no es el caso. Investigaciones recientes han avanzado en nuestro entendimiento de las influencias sociales, culturales y psicológicas en la toma de decisiones y el comportamiento que han demostrado tener un impacto significativo en los resultados del desarrollo" (World Bank, 2015, pág. xi).

El capítulo cuatro del reporte, correspondiente a la pobreza, menciona que adicional a los efectos descritos por Mani, et al (2013) en su investigación, la pobreza crea "marcos cognitivos pobres" o, en otras palabras, maneras de interpretar el mundo y el rol de las personas pobres en él, que los lleva a perder oportunidades de salir de la pobreza por un déficit de aspiraciones (World Bank, 2015).

Sheehy-Skeffington y Haushofer (2014) afirman que la pobreza inicialmente afecta la capacidad de control de los individuos sobre su entorno, esto se manifiesta en una disminución de la autoeficacia percibida. Esta falta de control desencadena tres características de la "mentalidad de pobreza", a saber, el estrés crónico, el agotamiento de recursos cognitivos debido a las preocupaciones monetarias, que genera una carga cognitiva y distrae a los individuos, incrementando la posibilidad de ceder ante heurísticos y sesgos; y, finalmente, la reducción de la capacidad cognitiva produce un estrechamiento atencional que lleva a tomar decisiones que a largo plazo perpetúan la situación de pobreza de las personas.

En las más recientes investigaciones sobre pobreza y funcionamiento cognitivo, autores como Schilbach, Schofield y Mullainathan (2016) señalan que el aumento de la carga cognitiva genera una especie de impuesto en el ancho de banda atencional, disminuyendo la capacidad cognitiva y el control ejecutivo. Una revisión de literatura sobre el tema muestra que la carga cognitiva lleva a peores juicios probabilísticos, reduce el desempeño en las tareas de predicción, incrementa la aversión al riesgo, lleva a las personas a asumir hábitos menos saludables, e incrementa el egoísmo (Deck \& Jahedi, 2015). 
Se hace evidente entonces que es necesario un cambio de enfoque si se quiere dar una solución de raíz a esta problemática. Darminger, et al (2015, pág. 14) plantean que es fundamental cambiar la pregunta de investigación sobre la pobreza de: “QQué hacen mal las personas que están en situación de pobreza, o qué barreras estructurales los están manteniendo en esa situación? a ¿Qué le está haciendo la pobreza a las personas, o qué características contextuales (acceso a servicios públicos, educación, salud, etc) están distorsionando sus elecciones y acciones?".

\section{Psicología y pobreza: perspectivas de investigación}

La pobreza sigue siendo uno de los grandes desafíos de las sociedades modernas, en Colombia, por ejemplo, 27,8\% de la población se encuentra en situación de pobreza y 7,9\% en situación de pobreza extrema (Departamento Administrativo Nacional de Estadísticas, 2016). Asimismo, según datos de las Naciones Unidas, 5,6\% de los habitantes del país viven con menos de USD $\$ 1,25$ al día (United Nations Development Programme, 2015).

La situación de pobreza tiene grandes impactos sobre la vida de los individuos que la sufren. Jiménez, Cuartas, Cristancho y Gómez (2016), a partir de un resumen de lo que reporta la literatura en el tema, muestran que estas personas sufren más inseguridad alimentaria, cuentan con menores dotaciones de capital humano (salud y educación), tienen más probabilidad de vivir en ambientes expuestos a mayor número de catástrofes naturales y conflictos armados, entre otros. Es evidente pues que, la pobreza se convierte en una trampa con bajas probabilidades de escape.

Ahora bien, como se señaló, las investigaciones desde la economía del comportamiento y la psicología han permitido identificar que la pobreza no solo genera carencias materiales, sino que también tiene consecuencias psicológicas (y biológicas), en palabras de Mullainathan y Shafir, citados por el Banco Mundial (2015, pág. 81)

"Si usted quiere entender a los pobres, imagínese a sí mismo con su mente en otra parte. No durmió mucho la noche anterior. Encuentra difícil pensar claramente. El autocontrol se siente como un desafío. Está distraído y fácilmente perturbado. Y esto pasa todos los días. Por encima de los otros desafíos materiales, la pobreza trae también un desafío mental... Bajo estas condiciones, todos hubiéramos (y lo hemos hecho) fallado.”

También se ha identificado que un bajo ingreso está altamente correlacionado con: bajas aspiraciones, fijación por las alternativas más cercanas en el tiempo (por la alta incertidumbre de vivir en situación de pobreza), aversión al riesgo, sentimientos de inutilidad de vivir, entre otros (Haushofer \& Fehr, 2014).

En la tabla 1 se presentan un conjunto de estudios que abordan los efectos de la pobreza sobre el funcionamiento psicológico. La revisión destaca la fuente consultada, los instrumentos para medir el impacto en las variables dependientes y el tamaño del efecto en la métrica de D de Cohen. En la sección de estudios se hace explícito si la condición metodológica es experimental o estudio de campo. Es importante señalar al respecto: (a) el carácter actual de la discusión sobre la pobreza en la literatura psicológica, que exhorta a redoblar esfuerzos en la investigación en el campo y (b) la estrategia de acercamiento metodológico a los efectos de la pobreza por dos vías, por una parte, con la participación de muestras con ingresos económicos bajos, y por otra, por la vía de la manipulación, es decir, a través de situaciones experimentales que fungen como versiones simuladas de las condiciones en las que se toman elecciones cuando se tiene ingresos económicos bajos. 
Tabla 1

Artículos de revisión de los efectos de cargas cognitivas de pobreza sobre el funcionamiento psicológico.

\begin{tabular}{|c|c|c|c|c|c|}
\hline Estudio & $\mathrm{N}$ & Dependiente & Estadístico & Valor & D de Cohen \\
\hline \multicolumn{6}{|l|}{ Mani et al, (2013) } \\
\hline Experimento 1 & 101 & Raven & $\mathrm{t}$ & 3,21 & 0.63 \\
\hline Experimento 1 & & Control Cognitivo & $\mathrm{t}$ & 5,22 & 1.03 \\
\hline \multirow[t]{2}{*}{ Experimento 3} & 100 & Raven & $\mathrm{t}$ & 3,34 & 0.66 \\
\hline & & Control Cognitivo & $\mathrm{t}$ & 3,54 & 0.7 \\
\hline \multirow[t]{2}{*}{ Experimento 4} & 96 & Raven & $\mathrm{t}$ & 3,55 & 0.72 \\
\hline & & Control Cognitivo & $\mathrm{t}$ & 3,34 & 0.68 \\
\hline \multirow[t]{3}{*}{ Estudio de campo } & 464 & Raven & Dif Med & 1.11 & \\
\hline & & Control Cognitivo (Velocidad) & Dif Med & $1.12 \mathrm{de}$ & 1 D.E. \\
\hline & & Control Cognitivo (Errores) & Dif Med & -7.7 & \\
\hline \multicolumn{6}{|l|}{ Shah et al, (2012) } \\
\hline Experimento 1 & 60 & Atención y Control Cognitivo & Eta2 & 0.07 & 0.54 \\
\hline Experimento 2 & 68 & Concentración & Eta2 & 0.17 & 0.9 \\
\hline Experimento 5 & 37 & Neglicencia atencional & Eta 2 & 0.03 & 0.35 \\
\hline \multicolumn{6}{|l|}{$\begin{array}{l}\text { Carvalho, Meier y } \\
\text { Wang, (2016) }\end{array}$} \\
\hline \multirow[t]{2}{*}{ Experimento 1} & 1056 & \multicolumn{4}{|c|}{ Función Cognitiva (memoria de trabajo) } \\
\hline & & \multicolumn{4}{|c|}{ Reflexión cognitiva } \\
\hline Experimento 2 & 2496 & Stroop & & $0.11 \mathrm{de}$ & $1 \mathrm{DE}$ \\
\hline \multicolumn{6}{|l|}{ Spears (2011) } \\
\hline \multirow[t]{2}{*}{ Experimento } & 57 & $\begin{array}{l}\text { Tiempo Extra Tensión Mancorna } \\
\text { (Control) }\end{array}$ & $\mathrm{z}$ & -0.72 & -0.19 \\
\hline & & Stroop & & & \\
\hline \multirow[t]{2}{*}{ Estudio de Campo } & 216 & Control (mancuerna) & $\mathrm{t}$ & -1.84 & -0.32 \\
\hline & & Memoria de trabajo & $\mathrm{X} 2$ & 3.04 & 0.23 \\
\hline
\end{tabular}

Fuente: Elaboración propia

A partir de este tipo de hallazgos, los gestores de política aceptaron, finalmente, un cambio en los mecanismos de lucha contra la pobreza. El nuevo marco de acción, que sugiere el Banco Mundial, se orienta a tres pilares fundamentales, a saber: (1) minimizar los costos cognitivos para las personas pobres, lo que implica simplificar los procedimientos, enfocarse en los más pobres, y reducir la volatilidad económica y mejorar la infraestructura, (2) evitar los marcos de pobreza, y, (3) incorporar el contexto social en el diseño de programas (World Bank, 2015). 
La revisión de literatura descrita evidenció que si bien ha habido un cambio en la aproximación a los estudios en pobreza y cada vez hay un interés mayor en explicar qué hace la pobreza a las personas, las variables cognitivas de análisis aún son muy limitadas (capacidad cognitiva y control ejecutivo), y su sustento teórico integrado es débil. Esto redunda en la existencia de un cuerpo empírico creciente, pero escasa comprensión de las causas teóricas que explican los efectos cognitivos, sociales, culturales, entre otros, de la pobreza.

Así pues, se proponen algunas líneas de investigación que pueden ampliar el aporte de la psicología a la lucha mundial contra este flagelo. Desde la perspectiva teórica, la psicología habrá de consolidar un marco teórico explicativo de los efectos cognitivos de la pobreza. Al respecto hay dos modelos teóricos prometedores: en primer lugar, los modelos de automaticidad (Hasher \& Zacks, 1979; Posner \& Snyder, 1975; Shiffrin \& Schneider, 1977), que permiten explicar los efectos de las tareas monetarias en la asignación de recursos atencionales, el desempeño cognitivo, y el funcionamiento de los procesos automáticos y controlados; y en segundo lugar, la teoría de la prospectiva (Kahneman, 2003), para analizar el efecto de las condiciones de pobreza sobre la toma de decisiones.

Estrechar la relación entre el desarrollo de los experimentos y la práctica teórica permite hacer inteligible en términos explicativos los hallazgos disponibles en variables cognitivas tales como la atención, la memoria, el funcionamiento ejecutivo y la toma de decisiones. Así, la psicología podrá abordar de forma sistemática la pregunta ¿Cuál es la influencia en los procesos cognitivos de los individuos al experimentar una situación de pobreza? Con un énfasis mayor en la situación y menor en la proveniencia socioeconómica de los participantes, abordando de forma analítica aspectos críticos para la comprensión del funcionamiento psicológico y la toma de decisiones económicas, por ejemplo el control inhibitorio, memoria de trabajo y atención; y en general aumentar la comprensión sobre los procesos automáticos y controlados - y sus interacciones -.

Por otra parte, es importante hacer un llamado a mantener el nivel de análisis propio de la disciplina psicológica en el estudio de la pobreza. Están bien establecidas las afectaciones que un ambiente empobrecido produce en el desarrollo cerebral de un amplio grupo de especies animales (Lipina \& Posner, 2012). Sin embargo, la pobreza no solo genera cambios neurofisiológicos en los individuos, también altera las demandas de recursos atencionales, restringiendo la capacidad de las personas para valorar los escenarios a los que se enfrentan, lo que los lleva con frecuencia a tomar peores decisiones debido a un menor control ejecutivo, mayor dificultad en la solución de problemas, entre otros (Mani, et al, 2013).

En este sentido, como lo señala Tomassini (2009), señalar que las afectaciones cerebrales inciden sobre el funcionamiento psicológico es verdadero, pero no todo en apariencia error en el funcionamiento psicológico implica un daño cerebral, o no en el sentido patológico. Este nivel de análisis, el funcionamiento psicológico y la relación con las condiciones contextuales habrá de mantenerse como práctica teórica y aporte diferencial en el trabajo interdisciplinario sobre pobreza.

Finalmente, vale la pena señalar que profundizar en este nivel de análisis también tiene implicaciones metodológicas para los estudiosos del tema. Así, la pobreza 
como variable de condición económica, ha de estudiarse de forma analítica y pertinente para el interés y alcance explicativo de la psicología. En términos del funcionamiento del sistema cognitivo, la pobreza más que predicarse de las características de un individuo, se predica de la interacción entre las demandas situacionales y los recursos cognitivos disponibles. Por tanto, la humanización de la decisión económica no es solo necesaria, también se puede privilegiar en los estudios y exigible en la publicación científica.

\section{Referencias}

Acemoglu, D., \& Johnson, S. R. (2006). Understanding Prosperity and Poverty: Geography, Institutions, and the Reversal of fortune. En A. Banerjee, R. Benabou, \& D. Mookherjee, Understanding poverty (págs. 19-36). New York: Oxford University Press.

Aghion, P., \& Armendáriz, B. (2006). A new growth approach to poverty alleviation. En A. Banerjee, R. Benabou, \& D. Mookherjee, Understanding poverty (págs. 7384). New York: Oxford Press University.

Banerjee, A., \& Duflo, E. (2007). The economics lives of the poor. Journal of economic perspectives, 21(1), 141 - 167.

Banerjee, A., Benabou, R., \& Mookherjee, D. (2006). Understanding poverty. New York: Oxford Press University.

Banerjee, A., \& Mullainathan, S. (2010). The shape of temptation: implications for the economic lives of the poor. Working Paper 15973. National Bureau of Economic Research. .

Banerjee, A., Duflo, E., Glennerster, R., \& Kinnan, C. (2013). The Miracle of Microfinance? Evidence from a Randomized Evaluation. MIT Department of Economics Working Paper No. 13-09.

Bertrand, M., Mullainathan, S., \& Shafir, E. (2004). A behavioral economics view of poverty. American Economic Review, 94(2), 419 - 423. Stable URL: http://www. jstor.org/stable/3592921

Bertrand, M., Mullainathan, S., \& Shafir, E. (2006). Behavioral economics and marketing in aid of decision making among the poor. Journal of Public Policy and Marketing, 25(1), 8 - 23. doi:10.1509/jppm.25.1.8

Calvo, A., \& Bialystok, E. (2014). Independent effects of bilingualism and socioeconomic status on language ability and executive functioning. Cognition, (130), 278 - 288. doi: 10.1016/j.cognition.2013.11.015

Camerer, C., \& Loewenstein, G. (2004). Behavioral economics: past, present, future. En C. Camerer, G. Loewenstein, \& M. Rabin, Advances in behavioral economics (pág. 725). Princeton: Princeton University Press.

Carr, S. C., Thompson, M., Dalal, A. K., de Guzman, J. M., Munns, A., \& Steadman, A. (2014). Psychology and poverty reduction: A global special issue. International perspectives in psychology: Research, practice, consultation, 3(4), 215-237. http:// dx.doi.org/10.1037/ipp0000021 
Carvalho, L. S., Meier, S., \& Wang, S. W. (2016). Poverty and Economic Decision-Making: Evidence from Changes in Financial Resources at Payday. The American Economic Review, 106(2), 260-284. http://doi.org/10.1257/aer.20140481

Curran, L. (2002). The psychology of poverty: professional social work and aid to dependent children in postwar America: 1946 - 1963. Social Service Review(Septiembre), 365-386. https://doi.org/10.1086/341180

Darminger, A., Hayes, J., Barrows, A., \& Wright, J. (2015). Poverty interupted: Applying behavioral science to the context of chronic scarcity. Ideas, 42.

Deck, C., \& Jahedi, S. (2015). The effect of cognitive load on economic decision making: A survey and new experiments. European Economic Review, (78), 97-119. https://doi.org/10.1016/j.euroecorev.2015.05.004

Departamento Administrativo Nacional de Estadísticas. (02 de Marzo de 2016). Departamento Administrativo Nacional de Estadísticas. Recuperado el 13 de Octubre de 2016, de https://www.dane.gov.co/files/investigaciones/condiciones_vida/ pobreza/bol_pobreza_15_.pdf

Dornbusch, R., \& Edwards, S. (1991). The macroeconomics of populism in Latin America. Chicago: University of Chicago Press.

Duflo, E. (2006). Poor but rational? En A. Banerjee, R. Benabou, \& D. Mookherjee, Understanding poverty (págs. 367-378). New York: Oxford University Press.

Graham, C. (1994). Safety nets, politics and the poor. Transitions to a market economy. Washington D.C: Brookings Institution.

Hall, C., Zhao, J., \& Shafir, E. (2013). Self-Affirmation among the poor: cognitive and behavioral implications. Psychological Science, 25(2), 619 - 625. doi: 10.1177/0956797613510949

Hasher, L., \& Zacks, R. T. (1979). Automatic and effortful processes in memory. Journal of Experimental Psychology, 108(3), 356. http://dx.doi.org/10.1037/00963445.108.3.356

Haushofer, J., \& Fehr, E. (2014). On the psychology of poverty. Science, 344(6186), 862 - 867. doi: 10.1126/science. 1232491

Jiménez, W., Jorge, C., Cristancho, J., \& Gómez, S. (2016). Algunas causas y consecuencias psicológicas de la pobreza: análisis entre países e individuos. Bogotá.

Kahneman, D. (1973). Attention and effort. New Jersey: Prentice Hall.

Kahneman, D. (2003). Mapas de racionalidad limitada: psicología para una economía conductual. Revista Asturiana de Economía, (28), 181 - 225.

Kahneman, D., \& Tversky, A. (1979). Prospect theory: an analysis of decisions under risk. Econometrica, 47(2), 263-291. http://www.jstor.org/stable/1914185

Katsnelson, A. (2015). News Feature: The neuroscience of poverty. PNAS, 112(51), 15530 - 15532. doi: 10.1073/pnas.1522683112

Katz, M. (1989). The undeserving poor: from the war on poverty to the war on welfare. New York: Pantheon.

Lipina, S., \& Posner, M. (2012). The impact of poverty on the development of brain networks. Frontiers in Human Neuroscience, 6, 1 - 12. doi: 10.3389/fnhum.2012.00238

Mani, A., Mullainathan, S., Shafir, E., \& Zhao, J. (2013). Poverty impedes cognitive function. Science, 341(6149), 976 - 980. doi: 10.1126/science.1238041 
McClelland, D. (1978). Managing Motivation to expand human freedom. American Psychologist, 33(3), 201. doi: http://dx.doi.org/10.1037/0003-066X.33.3.201

Mullainathan, S., \& Shafir, E. (2016). Escasez: ¿Por qué tener poco significa tanto? (Primera ed.). México D.F: Fondo de la Cultura Económica.

Noble, K., Norman, M., \& Farah, M. (2005). Neurocognitive correlates of socioeconomic status in kindergarten children. Developmental Science, 8(1), 74 - 87. doi: 10.1111/j.1467-7687.2005.00394.x

Posner, M. I., \& Snyder, C. R. (1975). Facilitation and inhibition in the processing of signals. Attention and performance, $V, 669-682$.

Raizada, R., Richards, T., Meltzoff, A., \& Kuhl, P. (2008). Socioeconomic status predicts hemispheric specialisation of the left inferior frontal gyrus in young children. Neuroimage, 40(3), 1392 - 1401. doi: https://doi.org/10.1016/j.neuroimage.2008.01.021

Sachs, J. (2006). The end of poverty: Economic possibilities for our time. Londres: Penguin Random House.

Schilbach, F., Schofield, H., \& Mullainathan, S. (2016). The Psychological Lives of the Poor. The American Economic Review, 106(5), 435-440.

Sen, A. (2015). Desarrollo y libertad (Décima tercera ed.). Bogotá: Planeta.

Shah, A., Mullainathan, S., \& Shafir, E. (2012). Some consequences of having too little. Science, 338(6107), 682 - 686. doi: 10.1126/science.1222426

Shah, A., Shafir, E., \& Mullainathan, S. (2015). Scarcity frames value. Psychological Science, 26(4), 402 - 412.

Sheehy-Skeffington, J., \& Haushofer, J. (2014). The behavioural economics of poverty. En I. I. Development, Barriers to and opportunities for poverty reduction: prospects for private sector - led interventions (pág. 176). Istanbul: United Nations Development Programme.

Shefrin, H. (2002). Beyond greed and fear. Understanding behavioral finance and the psychology of investing. New York: Oxford University Press.

Shiffrin, R. M., \& Schneider, W. (1977). Controlled and automatic human information processing: II. Perceptual learning, automatic attending and a general theory. Psychological Review, 84(2), 127. http://dx.doi.org/10.1037/0033-295X.84.2.127

Simon, H. A. (1972). Theories of bounded rationality. Decision and organization, 1(1), $161-176$.

Spears, D. (2011). Economic decision - making in poverty depletes behavioral control. The B.E. Journal of Economic Analysis \& Policy, 11(1), 1-40. doi: https://doi. org/10.2202/1935-1682.2973

Sunstein, C. (2000). Behavioral Law and Economics. Estados Unidos: Cambridge University Press.

Thaler, R. (2000). From Homo Economicus to Homo Sapiens. Journal of Economic Perspectives, 14(1), 133 - 141. http://www.jstor.org/stable/2647056

Tomassini, A. (2009) Ensayos de filosofía de la psicología (Tercera edición). Guadalajara: Universidad de Guadalajara

Turner, K., \& Lehning, A. (2007). Psychological theories of poverty. Journal of Human Behavior in the Social Environment, 16(1-2), 57-72. doi:10.1300/J137v16n01_05 
United Nations Development Programme. (2015). Human Development Report 2015. Washington: Human Development Report Office. Recuperado el 22 de Septiembre de 2016, de http://report.hdr.undp.org/

World Bank. (2015). World Development Report: Mind, society and behavior. Washington D.C: World Bank. 